\title{
Study On the Dynamic Modeling and the Correction Method of the Self-Propelled Artillery
}

\author{
Qicheng Zha ${ }^{1, \text { a }}$, Xiaoting Rui ${ }^{1, b^{*}}$, Feifei Liu ${ }^{2, c}$ and Hailong $Y u^{1, d}$ \\ ${ }^{1}$ Institute of Launch Dynamics, Nanjing University of Science and Technology, 200 Xiao Ling Wei, \\ Nanjing 210094, China \\ ${ }^{2}$ Inner Mongolia North Heavy Industry Group Limited Company, Nanjing branch, 200 Xiao Ling Wei, \\ Nanjing 210094, China.

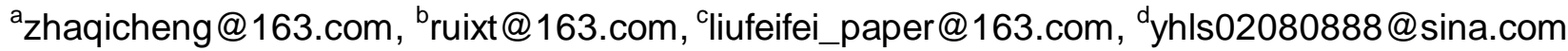 \\ ${ }^{*}$ corresponding author
}

Keywords: Mean point of impact; Average ballistic trajectory; Self-propelled artillery; Multi-body system dynamics; Multi-body system transfer matrix method; Firing correction.

\begin{abstract}
The artillery has been praised as "the god of war". Modern warfare requires the self-propelled artillery to have the ability to correct shots accurately. Most of the current fire control lacks of research in correct firing based on the mean point of impact. In order to improve the accuracy of the self-propelled artillery firing by use the mean point of impact, this paper established the launch and flight dynamics models of the self-propelled artillery by Multi-body System Transfer Matrix Method (MSTMM) and calculated the mean point of impact of the self-propelled artillery. The simulation results agree well with the experiment. The method of shooting correction based on the mean point of impact is established. The results provide a theoretical basis and technical means for improving the shooting accuracy of the self-propelled artillery.
\end{abstract}

\section{Introduction}

The artillery is an important fire strike force in modern warfare [1]. The self-propelled artillery is a ballistic bend, suitable for combat hidden targets and ground targets of field artillery, is one of the main gun species in ground artillery, by maneuvering can be divided into two kinds: traction and self-propelled. The new operational theory and new battlefield form put forward new requirements for self-propelled artillery effectiveness. The new operational theory puts forward the basic guiding ideology of the "main battle of firepower", which calls for carrying out a fire attack against the enemy in the full depth of the battle, and the proportion of the target in the depth of the enemy is obviously increased. In this case, it is difficult to deploy traditional reconnaissance force in the vicinity of the deep target. How to correct the gun firing quickly and accurately has great theoretical and practical significance, can greatly improve the operational effectiveness of artillery.

In case of unguided projectiles, to improve the weapon's accuracy [2], the error between the projectile impact point and target has to be estimated precisely to evaluate the weapon's accuracy and hence; an appropriate projectile firing correction is done based on the first shoot impact error to increase the next shoot accuracy. Traditionally, firing tables are used to calculate the standard projectile firing data needed to hit a desired target and also compute the firing data corrections in case of non-standard flight conditions [3]. These nonstandard flight conditions are resulted from variances in various parameters such as meteorological data, projectile mass properties, propellant temperature, and altitude difference between the launch point and the target [4]. To improve the weapon's hit accuracy, these nonstandard flight conditions have to be predicted accurately as well as possible especially the meteorological data [5],[6]. Using lookup search, all firing data can be determined automatically using a stored tabulated data for both standard and non-standard conditions. To speed up the searching process, different graphical tools such as graphical firing tables, graphical site tables, and wind cards, are used but with low accuracy due to simplifications [7]. 
In flight control systems using an iterative method, the solution of azimuth and quadrant elevation is determined through iterations of trajectory simulation in nonstandard conditions instead of searching firing data on the pre-calculated tabulated data in standard condition and then, applying corrections to compensate the corresponding nonstandard conditions [8]. NATO Armaments Ballistic Kernel (NABK) is one of the most notable systems uses an iterative method to compute the firing data [9], where the ballistic trajectory model used during iterations is for the modified point mass model (4-DOF) or five-degrees-of-freedom model [10]. Many algorithms were developed to ensure the firing data convergence during iterative trajectory simulations [11]. These iterative methods require an efficient algorithm to minimize the computation time.

In order to improve the performance of the fire control system, it is necessary to develop real-time correction technique for projectile external ballistics problems. Currently, firing correction methods are divided mainly into two kinds: (1) firing tables approximation; and (2) numerical methods. Due to the fast developments of computer technologies, numerical methods are widely used as the calculation processes become easier and general for any kind of gun, however, firing tables approximation is different for different gun type and different firing conditions. Many researchers developed different algorithms to improve the impact accuracy. In 1996, a mathematical model for determining firing data by computations considering the Coriolis effect and the firing site and the target were not in the same level was developed [12]. In 1997, a weighted function model based on small perturbation assumptions for all measurable real shooting conditions was developed to estimate the correction angle required to improve impact accuracy [13]. In 2001, a firing angle iterative range correction method for tank fire system was developed based on the point mass model [14]. Another correction method used in ship-borne gun fire system has been developed to calculate the fire data against the targets on shore [15]. In 2003, a model to calculate the firing data and flight time with the target position has been developed by solving the projectile external ballistic equations once only to reduce processing time, as well as methods to correct the informal ballistic and meteorological conditions, movement of the ship and the side deviation of projectiles were presented [16]. In 2007, Harlin and Cicci developed a state transition matrix STM to make corrections to ballistic missile initial state vector to achieve predefined final state vector using a 6DOF model [17]. In 2011, a differential algorithm with two height levels was developed to accurately calculating the ahead hit position for real antiaircraft gun system. In 2013, Marcelo Maritinez and Nicolas Bruno presented an analysis about the use of a new technology of optical strobe tracer in automatic firing weapons to improve ballistic solutions to fire control system using data from the projectile.

Recently, a number of optimization techniques have been used to improve fire control system performance, such as neural networks which is shorten the trajectory solver time but pre-trained neural network requires a lot of data and can not be generalized for different gun type, genetic algorithm GA, and particle swarm optimization algorithm PSO which is not only fast convergence but also simple implementation.

Many scholars have studied the correction of gun firing based on the return value of firing tables and miss distance. However, the previous artillery firing correction to a bullet firing point of the shooting as the basis for the next firing correction, but due to the initial disturbance of randomness, a bullet firing point can not reflect the mean point of impact of such firing weapons, may causes the correction to fail [18]. The actual firing correction should be based on the mean point of impact as the benchmark. Most of the current fire control lacks of research in correct firing based on mean point of impact.

After the projectile shot hit the target, the ground or airburst point (whether or not hitting the target) is called the point of impact. After launching the average position of the points of impact, called the mean point of impact. The average of a group of trajectories is called the average trajectory, as is shown in Fig. 1. 


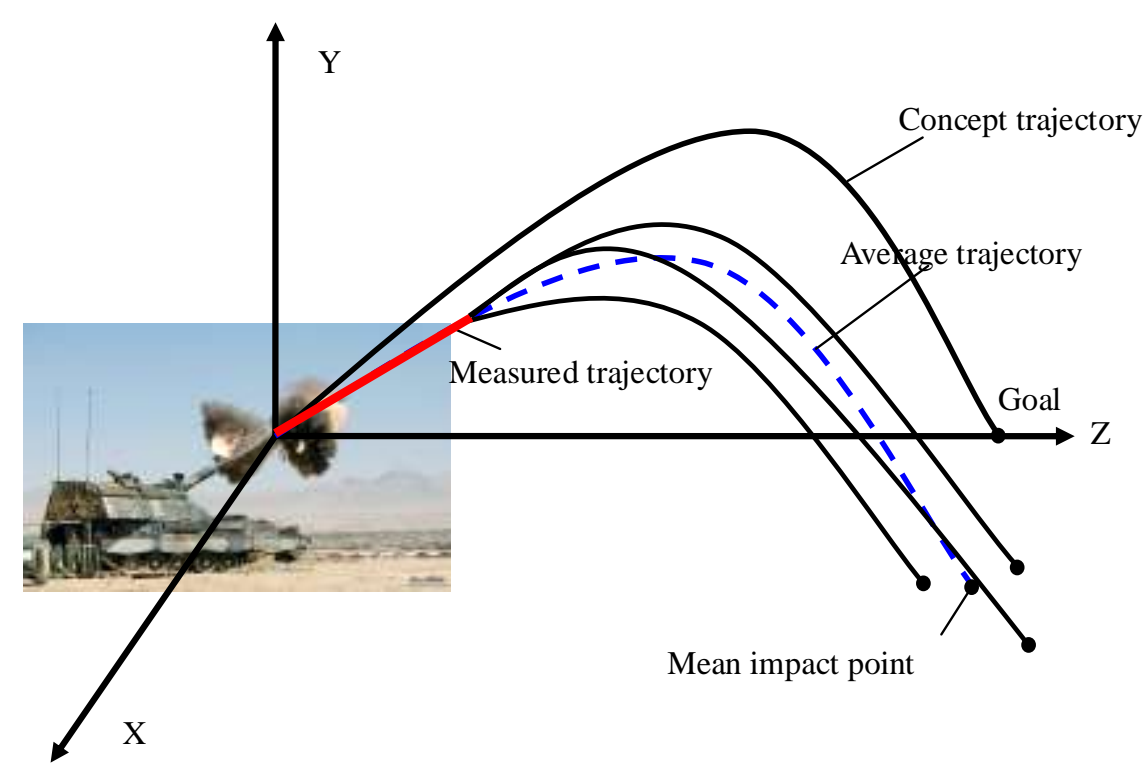

Fig. 1. Average trajectory diagram

In this paper, in order to obtain the average point of impact, improve self-propelled artillery firing accuracy, application by Multi-body System Transfer Matrix Method, The dynamics model of the self-propelled self-propelled artillery system, the dynamics model topology and the numerical simulation system are established. The accuracy of projectile shooting is calculated, and the average projectile is obtained. The calculated results agree well with the experimental results. In order to improve the accuracy of the self-propelled artillery firing accuracy, the angle correction formulas for the azimuth and quadrant elevation of the self-propelled artillery based on the average bullet point is established, corrected with satisfactory results.

\section{The Launch Dynamics Model of Self - Propelled Artillery System}

The M109A6 155mm type self-propelled artillery (USA) is shown in Fig. 2. Main components of self-propelled artillery are muzzle device, barrel (include evacuator), gun breech, recoil and counterrecoil mechanism, cradle, elevating mechanism, equilibrator, turret, traversing mechanism, chassis (artillery bogie), torsion bar, balance elbow, shock absorber, track chain, road wheel and so on.

According to the MSTMM [19], "bodies" are the units of motion, including rigid body and flexible body, "joints" are the links between bodies, including elastic joints, smooth joints, column joints, prismatic joints, fixed joints and so on. The masses of joints are considered in the contiguous bodies, so the joints are mass less, while the "bodies" and the "joints" are numbered uniformly.

Barrel part before the cradle is modeled as a flexible beam, numbered as 1; the rest part of the barrel is regarded as a rigid body, numbered as 3; the lifting part which doesn't contain recoil part is considered as a rigid body, numbered as 5; the masses of recoil and recuperator are considered in rigid bodies 5 and 3 , the effects of recoil and recuperator are equivalent to the elastic damping hinge and recoil resistance between gun breech and lifting part; the interaction between barrel and lifting part is equivalent to the slide hinge 4; Turret and hull are seen as rigid bodies, numbered as 7 and 9 respectively; the function of vertical aiming and stabilization are described by the control moment $M_{c \theta}$ acting on lifting part 5 and turret 7, the interaction between elevating mechanism and lifting part is equivalents to the column hinge 6; the function of lateral aiming and stabilization are described by the control moment $M_{c \alpha}$ acting on turret 7 and hull 9; the interaction between traversing mechanism and turret is equivalent to the column hinge 8 ; the engine is modeled as a rigid body, numbered as 49 ; the interaction between hull and engine along with the transmission system is equivalent to elastic damping hinges, which are numbered as 26 、 27、28、29; driving wheels are regarded as rigid bodies, numbered as 33 and 41; the interaction between driving wheels and hull are equivalent to elastic damping hinges 10 and 18; inducers are regarded as rigid 
bodies, numbered as 40 and 48; the elastic effect of inducers, the interaction between inducers and hull, and the interaction between inducers and tension devices are equivalent to elastic damping hinges 17 and 25 , respectively; the masses of torsion bar and shock absorber are considered in the hull, the equilibrium elbows are considered as rigid bodies, which are numbered as $34,35, \ldots, 39,42,43, \ldots, 47$; the interactions between the equilibrium elbows and the hull are equivalent to elastic damping hinges 11, $12, \ldots, 16,19,20, \ldots, 24$; the interactions between equilibrium elbows and load bearing wheels are equivalent to elastic damping hinges $53,54, \ldots, 64$; the 12 load bearing wheels are considered as rigid bodies, numbered as $65,66, \ldots, 76$; the track shoes are modeled as rigid bodies, the mass of the track pin is considered in its corresponding track shoe, the interaction between track shoes is equivalent to elastic damping hinge, then the track shoes on the left side are numbered as $77\left(\mathrm{n}_{1}\right) 、 77\left(\mathrm{n}_{3}\right) 、 77\left(\mathrm{n}_{5}\right) 、 \ldots$ $77\left(\mathrm{n}_{169}\right)$, and the hinges between the track shoes (on the left) are numbered as $77\left(\mathrm{n}_{2}\right) 、 77\left(\mathrm{n}_{4}\right)$ 、 $77\left(\mathrm{n}_{6}\right) 、 \ldots 、 77\left(\mathrm{n}_{170}\right)$, similarly, the track shoes on the right side are numbered as $78\left(\mathrm{n}_{1}\right) 、 78\left(\mathrm{n}_{3}\right)$ 、 $78\left(\mathrm{n}_{5}\right) 、 \ldots, 78\left(\mathrm{n}_{169}\right)$, and the hinges between the track shoes (on the right) are numbered as $78\left(\mathrm{n}_{2}\right)$ 、 $78\left(\mathrm{n}_{4}\right) 、 78\left(\mathrm{n}_{6}\right) 、 \ldots 、 78\left(\mathrm{n}_{170}\right)$; the three crews-seats are considered as rigid bodies, numbered as 50 、 51、52; the interaction between crews-seats and the hull are equivalent to elastic damping hinges 30,31 and 32. Therefore, the marching and launch dynamics model of the self-propelled artillery multi-body system is a complicated system which are composed of components of 207 bodies and 209 hinges. The dynamics model is shown in Fig. 3, and the topological graph of the dynamics model is shown in Fig. $4[20]$.

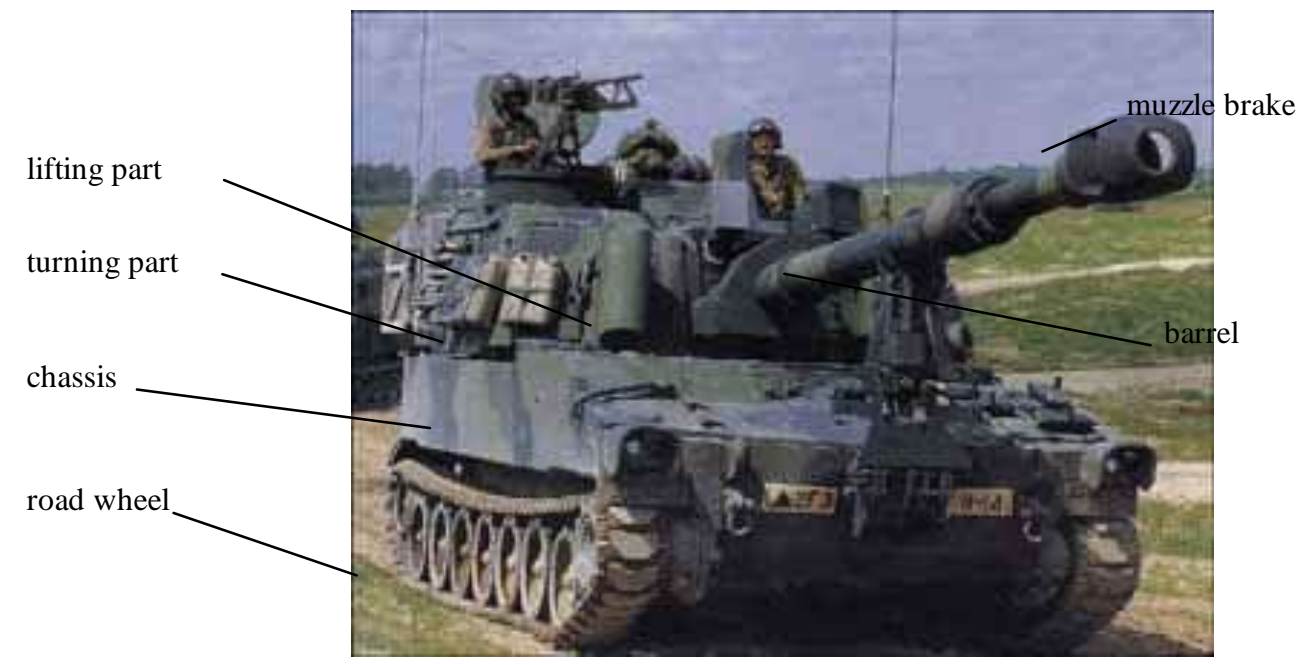

Fig. 2. US $155 \mathrm{~mm}$ self-propelled artillery

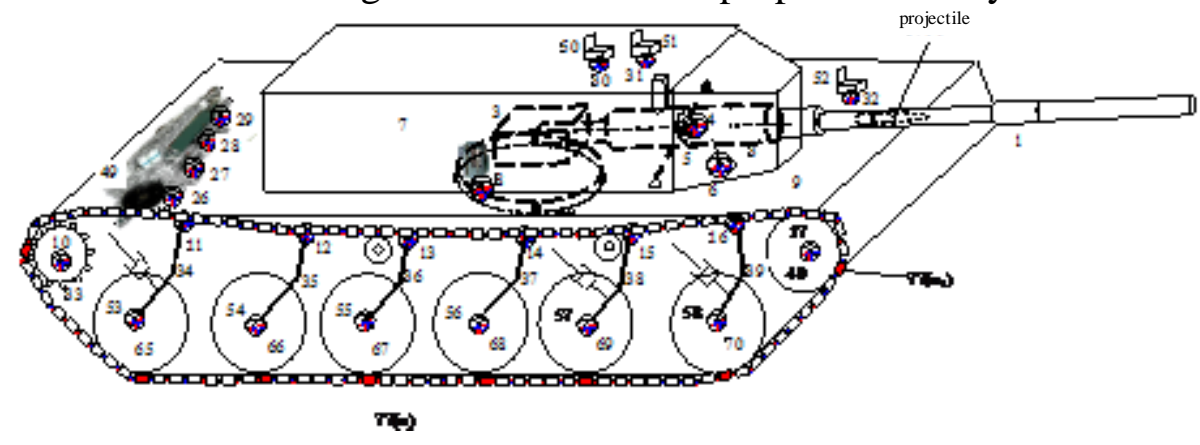

Fig. 3. Model of launch dynamics of the self-propelled artillery multi-body system 


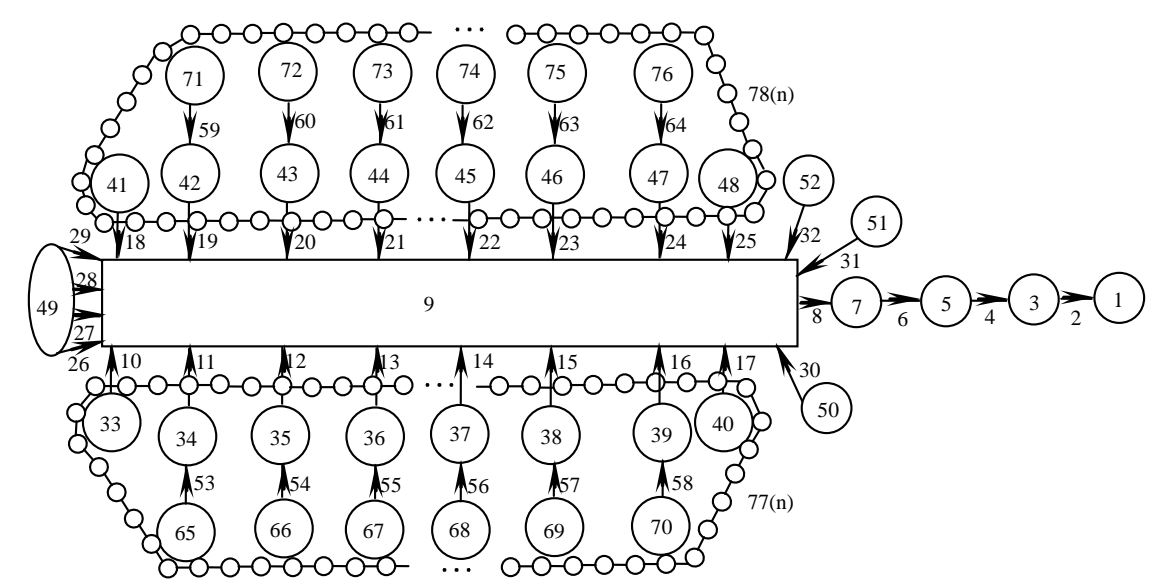

Fig. 4. Topological graph of launch dynamics of the self-propelled artillery multi-body system According to the multi-body system dynamics model, the topology structure of the self-propelled artillery, the automatic transfer theorem of the total transfer equation of the multi-body system, the total transfer equation of the self-propelled artillery multi-body system is

$$
U_{\text {all }} z_{\text {all }}=0
$$

Where, $\boldsymbol{U}_{\text {all }}$ is the total transfer matrix for the system, $\boldsymbol{z}_{\text {all }}$ is the system state vector. The linear acceleration, angular acceleration, internal torque and internal force at the connection point are described in the global inertial coordinate system.

The dynamic model of the self-propelled artillery can be obtained by using the multi-body system transfer matrix method. The dynamic response and the initial disturbance of the self-propelled artillery can be obtained by simultaneous launching dynamics equation. Then the initial disturbance is substituted into the $6 \mathrm{D}$ external ballistic equations and hence, get the shooting accuracy of the self-propelled artillery.

\section{The Simulation System of Self-Propelled Artillery Launch Dynamics}

By the book "Launch dynamics of tank and self-propelled artillery", the launch dynamics of the self-propelled artillery, the vibration characteristics and the simulation method of the six-degree-of-freedom external ballistics trajectory are studied in detail. The self-propelled artillery system is launched and the flight dynamics numerical simulation system, the simulation flow chart is shown in Fig. 5. 


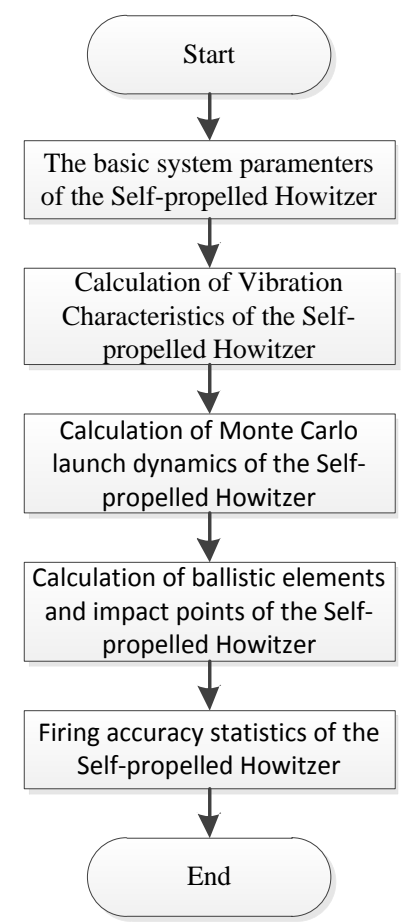

Fig. 5. Flow chart of a self-propelled artillery system launch and flight dynamics simulation system

\section{The Impact Point Correction System of Self-Propelled Artillery}

The correction amounts of the gun impact point is the correction amounts of the data at corresponding firing point. Including the correction amount of range, the correction amount of drift and the correction amount of blast-target vertical interval. There are three main methods for correcting the firing amounts of the impact point correction: adjusts the elevation angle, adjusts the azimuth angle and adjusts the amount of charge. The main method of reducing the correction amount of range is divided into adjusts the charge in order to adjusts the muzzle velocity and adjusts the elevation angle to control the impact point. The main method of reducing the correction amount of drift is adjusting the azimuth angle. The main method of reducing the correction amount of blast-target vertical interval is divided into adjusts the charge in order to control the muzzle velocity and adjusts the elevation angle to control the impact point (Wang et al 2012).

In this paper, we consider the correction of the range in the longitudinal $X$ direction and the drift in the transverse $\mathrm{Z}$ direction on the ground coordinates XOY of the same ground plane.

Correcting the firing of the self-propelled artillery by the deviation of the mean point of impact relative to the target point. The range adjusts the elevation angle and the drift adjusts the azimuth angle. Use a large number of Monte Carlo simulations, so that the difference between the target point and the mean point of impact satisfies the error. At this time of the firing element as a standard, to improve the accuracy of shooting purposes.

1) Range: Elevation angle can be adjusted by using Newton-Raphson method for the iterative calculation.

If the average rate of change $\frac{f\left(\theta_{k}\right)-f\left(\theta_{k-1}\right)}{\theta_{k}-\theta_{k-1}}$ of function $f\left(\theta_{k}\right)$ is used to approximate the instantaneous rate of change $f^{\prime}\left(\theta_{k}\right)$, the iterative format for the elevation angle that converges to the mean point of impact is:

$$
\theta_{k+1}=\theta_{k}-\left(f\left(\theta_{k}\right)-f\left(\theta_{M}\right)\right) \cdot \frac{\theta_{k}-\theta_{k-1}}{f\left(\theta_{k}\right)-f\left(\theta_{k-1}\right)}
$$


Where, $\theta_{k-1} 、 \theta_{k}$ is the elevation value at the begin of the iteration, $\theta_{k}=\theta_{k-1}+\Delta \theta, \Delta \theta$ is the iteration step at the begin of the iteration, $f\left(\theta_{k-1}\right) 、 f\left(\theta_{k}\right) 、 f\left(\theta_{M}\right)$ is the corresponding range of elevation angle $\theta_{k-1}, \theta_{k}$ and the mean point of impact M.

2) Drift: Azimuth angle can be adjusted by the angle between two vectors of the target point and the impact point of the final iteration as follows,

$$
\angle A O T=\arccos \left(\frac{\overrightarrow{O A} \bullet \overrightarrow{O T}}{|\overrightarrow{O A}| \cdot|\overrightarrow{O T}|}\right)
$$

Where, $\mathrm{A}$ is the impact point of changed the elevation angle, $\mathrm{O}$ is the origin point of the coordinate, $\mathrm{T}$ is the target point, $\angle A O T$ is the correction to the azimuth.

The correction specific steps of elevation and azimuth angles are as follows:

(a) Input the projectile data, aerodynamic data, wind data, and initialize the muzzle firing parameters;

(b) Given elevation and azimuth, use Monte Carlo launch dynamics calculation and six degree of freedom ballistic equations to get the mean point of impact;

(c) Determine the value of the elevation step and calculate the corresponding range $f\left(\theta_{k-1}\right), f\left(\theta_{k}\right)$ of iteration starting point $\theta_{k-1}, \theta_{k}$, carry on calculation of the elevation iterative, the iteration of the elevation angle is ended when the error of the iteration of the range is less than the given error;

(d) The azimuth angle calculation is carried out. When the iteration error of the drift is less than the given error, the iteration of the azimuth angle is ended.

(e) Record the correction of elevation and azimuth, analyzes the error situation.

The flow chart of elevation and azimuth angle correction algorithm is shown in Fig. 6.

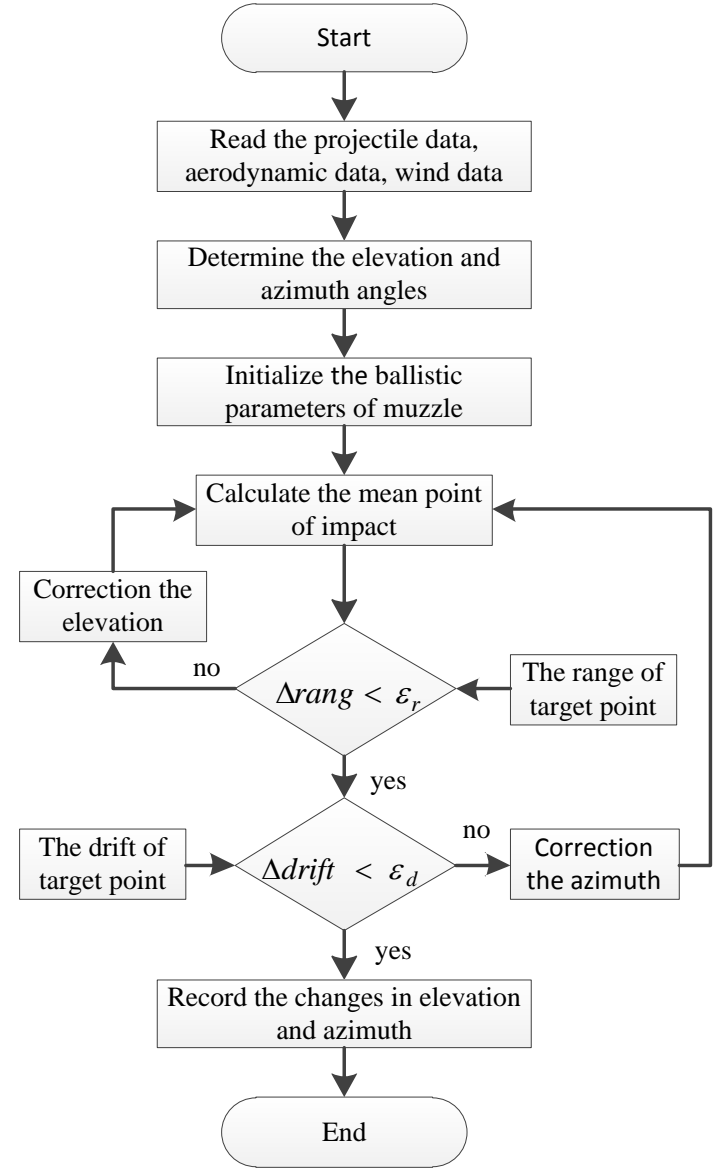

Fig. 6. Flow chart of elevation and azimuth angle correction algorithm 
In the experiment, The elevation and azimuth angles are $\left(45.0^{\circ}, 3.0^{\circ}\right)$, the coordinates of the target point are $(30000 \mathrm{~m}, 2500 \mathrm{~m})$, through the range and the drift difference between the mean point of impact and the target point, into the elevation and the azimuth angle iterative formulas, and select the appropriate initial iteration step, the correction values of elevation and azimuth are obtained. The result of the iteration is shown in Table 1.

Table 1 The Table of Correction for The Mean Point of Impact to The Target Point

\begin{tabular}{ccccc}
\hline Iteration & Range, $m$ & Drift, $m$ & Elevation, deg & Azimuth, deg \\
\hline 1 & 29925.7005 & 2485.2738 & 48.1151 & 3.0 \\
2 & 29972.7913 & 2513.6780 & 48.8176 & 3.0 \\
3 & 29992.9355 & 2528.5359 & 49.1872 & 3.0 \\
4 & 29996.8868 & 2531.7677 & 49.2686 & 3.0 \\
5 & 29999.3357 & 2499.8345 & 49.2686 & 2.6892 \\
\hline
\end{tabular}

The mean point of impact after correction is $(29999.3357 \mathrm{~m}, 2499.8345 \mathrm{~m})$, the corresponding elevation and azimuth angles are $\left(49.2686^{\circ}, 2.6892^{\circ}\right)$. The elevation and azimuth corrections are $\left(4.2686^{\circ},-0.3108^{\circ}\right)$. Effectively improving the self-propelled artillery firing accuracy.

\section{Conclusion}

In this paper, in order to improve the firing accuracy of the self-propelled artillery, from the perspective of self-propelled artillery system launch and flight dynamics, the multi-body system dynamics model and its topological structure of self - propelled artillery are established. The simulation results agree well with the experimental results. A modified iteration based on the mean projectile point is carried out. The results show that the method can effectively improve the accuracy of the self-propelled artillery firing. It provides a theoretical basis and technical means for the self-propelled artillery firing correction and improving shooting accuracy.

\section{Acknowledgements}

The research was Supported by Science Challenge Project (JCKY2016212A506-0104), the Natural Science Foundation of China Government (11472135), and the Research Fund for the Doctoral Program of Higher Education of China (20133219110037).

\section{References}

[1] X.T. Rui , L. F Yun., and Y.Q. LU. Transfer matrix method of multi-body system and its applications (Science Press, China 2008). (In Chinese)

[2] W. X. Shi., J.P. Qian and C. Fu. "Simulating the Effectiveness of Naval Gun Fire on the Sea by Monte Carlo Method.” J. Journal of Sichuan Ordnance, Vol 32 (2011) No.6, p.14-17. (In Chinese)

[3] E. R. Dickinson.The production of firing tables for cannon artillery. BRL Report 1371, Ballistic Research Laboratory, MD, USA. (1967)

[4] P. Chusilp, W. Charubhun and N. Nutkumhang. "Investigating an iterative method to compute firing angles for artillery projectiles." J. The 2012 IEEE/ASME International conference on Advanced Intelligent Mechatronics, Kaohsiung, Taiwan, (2012) July 11-14, p.940-945.

[5] B.R. Hansen, S. Aytarortac and A.J. Sowa. "NATO testing in Turkey show benefit of meteorological forecast data to indirect fire support." J. $24^{\text {th }}$ International Symposium on Ballistics, New Orleans, LA, USA, (2008) September, 22-26. 
[6] T.C. Jameson, S.A. Luces and D. Knapp. "Comparisons of ballistic trajectory simulations using artillery meteorological messages derived from local balloon data and battlescale forecast model data for the 1998 SADARM IOT\&E firings.” ARL-TR-1018, Army Research Laboratory, MD, (2001)USA.

[7] J.A. Matts and D.H. McCoy. "A graphical firing table model and a comparison of the accuracy of three utilization schemes." BRL Report No. 2035, Ballistic Research Laboratory, MD, (1970)USA.

[8] P. Chusilp, W. Charubhun and A. Ridluan. "Developing firing table software for artillery projectile using iterative search and 6-DOF trajectory model." J. The Second TSME International Conference on Mechanical Engineering, Krabi, Thailand, (2011)October 19-21.

[9] A.J. Sowa. "NATO shareable software developing into true suite supporting national operational fire control systems." J. $24^{\text {th }}$ International Symposium on Ballistics, New Orleans, LA, USA, (2008)September 22-26.

[10]P. Zipfel. Modeling and Simulation of Aerospace Vehicle Dynamics, 2nd edition, AIAA, USA. (2007)

[11] S.A. Ortac, U. Durak and U. Kutluay.. "NABK based next generation ballistic table toolkit." J. 23 ${ }^{\text {rd }}$ International Symposium on Ballistics, Tarragona, Spain, (2007)April 16-20.

[12] Y. Liu and Q. Yang. "A Mathematical Model of Determining Firing Data By Computer." J. Journal of Ballistics. Vol 8 (1996) No.3, p.66-68(In Chinese)

[13]X. Xue and X. Liu (1997). "Weighted Function Method For Exterior Ballistic Model In Fire Control System.” J. Acta Armamentarii ,.Vol 18 (1997) No.3, p. 234-238. (In Chinese)

[14] Q. Zhou, L. Yu and X. Giu . "Research on Real-Time Ballistic for Battle Vehicles." J. Fire Control and Command Control. Vol 26 (2001) No.4, p. 15-18. (In Chinese)

[15] H. Pan, J. Hu, S. Yang and D. Wang. "Method to Calculate the Ship-borne Gun Fire Data Against Shore." J. Fire Control and Command Control, Vol 26 (2001) No.4, p. 51-53. (In Chinese)

[16] H. Pan, J. Hu, S. Yang and D. Wang. "Model For Finding Firing Data Of Ship Borne Gun Weapon System By Solving External Ballistic Equations.” J. Acta Armamentarii, Vol 24 (2003) No.1, p. 23-26. (In Chinese)

[17] W. J. Harlin and D. A. Cicci. "Ballistic missile trajectory prediction using a state transition matrix." J. Applied Mathematics and Computation, Vol 188 (2007) No.2, p. 1832-1847.

[18]X. T. Rui, Q. R. Yang. (1991). Launch process of projectile, (South East University press, China 1991). (In Chinese)

[19]X. T. Rui, J. S. Zhang, Q. B. Zhou. "Automatic deduction theorem of overall transfer equation of multibody system." J. Advances in Mechanical Engineering, Article ID 378047, 12 pages, (2014). (In Chinese)

[20]F. Liu X. Rui and H. YU. Study on Launch Dynamics of the Tank Marching Fire, J. Journal of shanghai Jiaotong University (Science), Vol 21 (2016) No.4, p. 443-450. (In Chinese) 